\title{
Estudo comparativo sobre a composição e a diversidade de lepidópteros noturnos em cinco áreas da Região dos Cerrados ${ }^{1}$
}

\author{
Amabílio J. Aires de Camargo ${ }^{2}$
}

\begin{abstract}
Comparative study on composition and diversity of the moths in five areas of Cerrado Region. The understanding of the distribution and richness patterns of species is very important in ecology. Also, for the Cerrado region, this is very important and urgent, because of the strong pressure for new areas designated for agriculture. In five areas of the Cerrado, an exploratory study was carried out on the diversity, evenness and similarity of moth species. Moths were captured in a white sheet hung vertically and illuminated by a 250 Watt mercury vapor lamp. The alpha diversity was calculated using the Shannon-Wiener index ( $\left.\mathrm{H}^{\prime}\right)$ and the Simpson index $(\gamma)$. The beta diversity was examined for all sampled localities and estimated by Wittaker's measure. The Sörensen and Jaccard indexes were used to calculate similarity. A total of 11130 individuals, 1064 species and 33 families were collected during the study. Great differences were found in the diversity ( $\left.\mathrm{H}^{\prime}\right)$, and according to this index, the minimum value found was 1.60 and highest value was 4.59 . From the Simpson index $(\gamma)$, the minimum value was 0.42 and highest 0.96 . With both methods, in all the localities, these values were significantly different by the $T$ test $(p<0.001)$. The mean beta diversity found was 0.6929 , and the minimum and highest values found were respectively 0.4328 and 0.8615 . A positive correlation was found between beta diversity and distances among the localities $\left(r^{2}=0.5844, p<0.05\right)$. Faunal similarities among sites were low, and the highest value found was 0.4146 by Sörensen index and 0.2615 by Jaccard index.

KEY WORDS. Lepidoptera, moths, Brazilian savannas, biodiversity, similarity.
\end{abstract}

O Cerrado, concebido como um bioma, constitui-se no segundo maior domínio morfoclimático do Brasil e da América do Sul, ocupa 22\% do território brasileiro, perdendo em área apenas para a Floresta Amazônica (AB'SABER 1977, 1983).

O clima da região é tropical, com distribuição fortemente estacional das chuvas. Apresenta em sua maior porção duas estações bem definidas: uma chuvosa (outubro a abril), e outra seca (maio a setembro). A precipitação média anual varia entre $600 \mathrm{~mm}$ e $2200 \mathrm{~mm}$, e a altitude de $300 \mathrm{~m}$ a $900 \mathrm{~m}$ em mais de $70 \%$ da região, AB'SABER (1983) e ADÁMOLI et al. (1986), com temperaturas médias anuais variando entre $22^{\circ} \mathrm{C}$ a $28^{\circ} \mathrm{C}$ em $80 \%$ da área (DIAS 1992).

A região é constituída de grande número de habitats naturais com gradiente ambiental variando desde formações vegetais abertas, como os Campos Graminosos e as Veredas, até formações mais fechadas como os Cerradões, Matas Mesofíticas

1) Projeto financiado pela Japan International Cooperation Agency, através da Companhia de Promoção Agrícola.

2) Embrapa Cerrados. Caixa Postal 08223, 73301-970 Planaltina, Distrito Federal, Brasil.

E-mail: amabilio@cpac.embrapa.br 
e as Matas Ciliares (ALHO 1994). Essas diversificadas condições ecológicas abrigam vasto conjunto de comunidades, na sua maioria pouco conhecidas, principalmente no que se refere à sua fauna.

A perda da biodiversidade é, hoje, questão central nos principais fóruns de discussão sobre ecologia, e no caso dos Cerrados brasileiros, esta diversidade biológica está ainda na fase de caracterização para vários grupos, principalmente o dos insetos. A região, no entanto, já se encontra incorporada ao processo de produção de grãos, e a conversão da sua vegetação nativa em áreas agrícolas, tem sido uma das principais vias de degradação da sua biodiversidade (RIBEIRO \& SILVA 1996).

Com respeito à conservação da sua biota, uma das questões importantes que vem sendo abordada, diz respeito à exclusividade da sua fauna e flora. Existe um consenso de que, pelo menos para certos grupos de vertebrados, ao contrário da vegetação, não se pode falar de uma fauna endêmica, visto que, a maioria dos animais encontrados no domínio dos Cerrados, são espécies de ampla distribuição com ocorrência também em outras regiões (VANZOLINI 1963; SICK 1965; REDFORD $\&$ FONSECA 1986).

Para os vertebrados, a fauna que habita os Cerrados é portanto, compartilhada com outras formações abertas da América do Sul e apresenta-se fortemente influenciada pela Mata Atlântica e Floresta Amazônica e em menor escala pela Caatinga e Chaco, possuindo poucos endemismos (VANZOLINI 1963; SICK 1965; MARINHOFiLHo 1992; MARINHO-FILHO et al. 1994; SiLva 1995a,b). Em relação aos invertebrados, são poucos os estudos existentes que permitem uma análise biogeográfica para os Cerrados. No entanto, esse padrão de baixo endemismo, com forte influência da Mata Atlântica e Floresta Amazônica constatado para outros grupos é certamente válido para Saturniidae (Lepidoptera).

Embora o número total de espécies de Lepidoptera seja desconhecido para o Cerrado, Brown \& MielKe (1967a,b) e MielKe (1968) sugerem 984 espécies diurnas para o Planalto Central do Brasil. Para as espécies noturnas, das 400 espécies de Saturniidae, estimadas para o Brasil, $42 \%$ delas têm ocorrências registradas na região dos Cerrados. Estimativas feitas por V.O. Becker (comunicação pessoal) indicam que o número total de espécies com hábito noturno para toda região dos Cerrados pode ser superior a 10.000 .

A descrição e compreensão dos padrões de distribuição e riqueza são fundamentais em ecologia. No caso dos Cerrados, a forte pressão do homem por novas áreas, principalmente destinadas à agricultura, tornam estes estudos ainda mais urgentes.

A heterogeneidade da região, constatada por vários autores: CosTA et al. (1981); PINHEIRO \& ORTIZ (1992); RATTER \& DARGIE (1992); FELFILI et al. (1994); MARINHO-FILHO et al. (1994); Silva (1995a); RATTER et al. (1996), ilustra os cuidados que devem ser tomados na definição de áreas para preservação.

Este trabalho tem como principal objetivo analisar comparativamente a composição e a diversidade de lepidópteros noturnos, em cinco áreas da região dos Cerrados, dando subsídios para futuros estudos sobre a ecologia e distribuição do grupo. 


\section{MATERIAL E MÉTODOS}

As condições de amostragem para este estudo refletem a situação a que está submetida a maior parte da região dos Cerrados atualmente, isto é, ilhas de preservação ao meio de extensas áreas antrópicas, principalmente com lavouras de monoculturas. Mesmo as unidades de conservação como os Parques Nacionais, Reservas e Áreas de Proteção, estão sujeitas às pressões do homem, sendo o Cerrado, hoje, grande mosaico de ambientes com diferentes graus de manejos, permeado por pequenas áreas com vegetação nativa.

O estudo foi realizado em áreas preservadas de cerrado lato sensu, pertencentes a projetos de colonização do PRODECER (Programa de Desenvolvimento dos Cerrados). As cinco áreas pesquisadas compreendem o projeto de colonização Mundo Novo no município de Paracatu, Minas Gerais $\left(17^{\circ} 13^{\prime} \mathrm{S}, 46^{\circ} 52^{\prime} \mathrm{W}\right)$, com altitude de $920 \mathrm{~m}$ e área preservada contínua de 2360 ha; Projeto de colonização

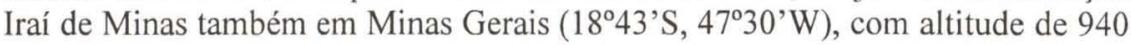
m e área preservada não contínua de 1800 ha, ambas amostradas em fevereiro de 1994. O terceiro e quarto locais de estudo foram no projeto de colonização Ouro Verde, com altitude de $700 \mathrm{~m}$, área preservada contínua de 800 ha e projeto Brasil Central, com altitude de $720 \mathrm{~m}$ e área preservada contínua de 2500 ha, ambas localizadas no município de Formosa do Rio Preto, no Oeste do estado da Bahia $\left(11^{\circ} 03^{\prime} \mathrm{S}, 45^{\circ} 12^{\prime} \mathrm{W}\right)$. As áreas da Bahia foram amostradas em dezembro de $1994 \mathrm{e}$ janeiro de 1995. O último ponto de estudo foi o projeto de colonização Gerais de Balsas no município de Balsas, Sul do estado do Maranhão (08 $38^{\prime} \mathrm{S}, 46^{\circ} 43^{\prime} \mathrm{W}$ ), com altitude de $525 \mathrm{~m}$, área preservada contínua de 2585 há, amostrada em janeiro de 1996. Em todas as áreas estudadas a soja foi a monocultura mais próxima, embora outras culturas agrícolas também estivessem presentes a uma distancia maior das reservas.

Foram utilizadas armadilhas luminosas que consistem em dois tecidos brancos, suspensos vertical e perpendicularmente, medindo dois metros de comprimento por um metro de largura. Para atrair os insetos foram usadas lâmpadas mistas de 250 watts, alimentadas com gerador à gasolina (Fig. 1). O critério principal na disposição de montagem das armadilhas foi sempre a direção do vento, isto é, um dos tecidos foi instalado em sua direção, na ausência deste a disposição foi aleatória, obedecendo apenas a mesma direção para todas as armadilhas instaladas naquela noite.

O esforço de captura empregado em cada área foi de seis noites no horário compreendido entre 20 e 21 horas. Foram escolhidas noites sem chuva e pouco vento, com fases lunares nova ou minguante.

SiLVEIRA NETO et al. (1976), demonstraram que existe uma periodicidade vôo quando se coleta insetos com armadilhas luminosas. Estes autores determinaram a hora de vôo de três mariposas da família Noctuidae, Agrotis ipsilon (Hufnagel, 1766), Helicoverpa zea (Boddie, 1850) e Spodoptera frugiperda (J.E. Smith, 1797), cujos resultados mostram que entre 18 horas e 21 horas ocorre o maior pico do número de indivíduos, entre 21 horas e 24 horas este número cai cerca de $40 \%$ em média, e depois desse horário o número de indivíduos cai drasticamente para menos de $10 \%$ em qualquer uma das três espécies estudadas. Como não é conhecido o 


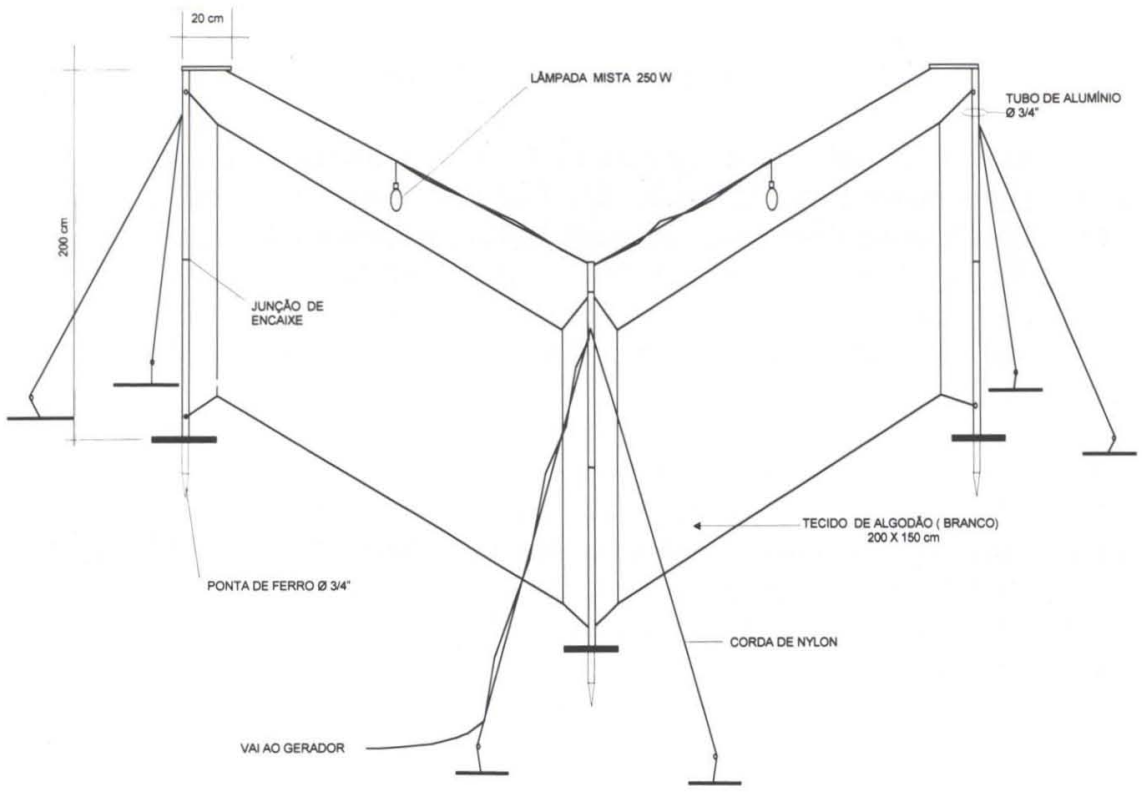

Fig. 1. Modelo de armadilha luminosa utilizada.

comportamento da maioria das espécies, optou-se por fazer as coletas para este estudo no horário de maior pico destas espécies.

O período de uma hora de coleta foi definido de acordo com THOMAS \& THOMAs (1994), o qual indica que para análises de diversidade ecológica de lepidópteros noturnos, a coleta por um período de uma hora por noite apresenta valores semelhantes a uma coleta de oito horas, apresentando a vantagem de diminuir os efeitos negativos de retirada da população, além de apresentar menor quantidade de material que deverá ser analisado no dia seguinte.

Todos os indivíduos atraídos pela armadilha foram capturados, os microlepidópteros em tubos de ensaio, os macrolepidópteros em câmaras mortíferas convencionais contendo amônia.

O material entomológico foi separado, etiquetado, e identificado pelo autor com auxílio do Dr. Vítor O. Becker ao nível de famílias e morfoespécies. Os espécimes examinados, (11.130), que correspondem a 1064 morfoespécies, encontram-se depositados na coleção da Embrapa Cerrados, acondicionados em gavetas entomológicas.

\section{Análise de diversidade}

Com a finalidade de avaliar a diversidade ecológica alpha $(\alpha)$ entre as comunidades e para fazer comparações entre elas, foram utilizados os seguintes índices: de Shannon-Wiener (MAGURRAN 1988), que varia de 0 a Ln do número de espécies amostradas, determinados pelo número de espécies presentes na comunidade e pela base da escala logarítmica escolhida. $\mathbf{H}^{\prime}=-\Sigma$ pi.Lnpi onde: $\left(\mathrm{H}^{\prime}\right)$ índice de diversidade de Shannon-Wiener, (Ln) logaritmo neperiano; (pi) ni/N, (ni) número de indivíduos amostrados de cada espécie, $(\mathrm{N})$ número total de indivíduos. 
O outro índice utilizado foi o de Simpson (MARGALEF 1991) que varia de 0 a 1 , e sua fórmula é $\gamma=1-\Sigma(\mathrm{ni} / \mathrm{N})^{2}$ em que "n" representa o número de exemplares de cada espécie e "N" o número total de exemplares na amostra, neste caso, o valor 1 equivale ao máximo de diversidade.

A diversidade beta ( $\beta$ ), que é essencialmente uma medida de comparação entre habitats, em termos da composição de espécies, a qual se baseia em dados de presença e ausência, foi calculada com base no índice de WHITTAKER (1960). Para cálculo dos valores de $(\beta)$ foi utilizada a fórmula $\beta^{\mathbf{w}}=(\mathbf{S} / \alpha)-\mathbf{1}$, em que $S$ é o número total de espécies coletado nas áreas consideradas, e $\alpha$ representa a diversidade média da amostra (riqueza média entre os pares de localidades comparadas). Por este índice, o valor 1 representa completa dissimilaridade, e zero indica que as comunidades não são diferentes na composição de espécies. As distâncias entre as localidades foram calculadas com base em mapa de escala 1:100.000, levando-se em conta a menor distância entre elas, isto é, traçado em linha reta.

\section{Análise de similaridade}

Foram feitas comparações qualitativas baseadas na presença e na ausência das espécies de acordo com dois índices: SÖRENSEN (1948) CS= 2c/a+b e JACCARD (1912), citado por Silveira Neto et al. (1976), onde $\mathbf{C J}=\mathbf{c} / \mathbf{a}+\mathbf{b}-\mathbf{c}$, sendo: (a) número de espécies da comunidade $\mathrm{A}$; (b) o número de espécies da comunidade $\mathrm{B}$, (c) representa o número de espécies comuns as duas comunidades. Ambos variam de 0 a 1 , sendo que 0 valor 1 representa o máximo de semelhança.

\section{RESULTADOS E DISCUSSÃO}

\section{Caracterização da entomofauna}

Ao todo foram examinados 11.130 exemplares, distribuídos entre 1064 espécies pertencentes a 33 famílias.

Das 33 famílias reconhecidas, 20 delas, não obrigatoriamente as mesmas, estiveram representadas em Iraí de Minas, Paracatu e Balsas, 17 no Projeto de colonização Ouro Verde (Formosa do Rio Preto, Bahia) e 26 no projeto agrícola Brasil Central, também no município de Formosa do Rio Preto. O número total de espécies, famílias e indivíduos, para cada uma das áreas estudadas é mostrado na tabela I.

Nas cinco comunidades pesquisadas, as espécies apresentam-se distribuídas diferenciadamente entre às famílias, sendo que a maior percentagem de espécies em todas as áreas são pertencentes as famílias Noctuidae e Pyralidae. Estas duas famílias somadas foram responsáveis por $57.78 \%$ a $75.58 \%$ de contribuição nas diferentes comunidades.

A participação das espécies da família Noctuidae variou de $33.24 \%$ em Iraí de Minas a 50\% em Formosa do Rio Preto (Projeto Ouro Verde). As espécies da família Pyralidae contribuíram com valores que variou de $18.35 \%$ em Formosa do Rio Preto (Projeto Brasil Central) a 28.29\% em Paracatu. As outras famílias com maior número de espécies foram Geometridae, Arctiidae, Gelechiidae e Oecophoridae, embora tenha havido variação quantitativa entre as áreas (Tab. II). 
Tabela I. Número de famílias, espécies e indivíduos de Lepidoptera coletados com armadilha luminosa em cinco áreas da região dos Cerrados.

\begin{tabular}{|c|c|c|c|}
\hline Localidades & Número de familias & Número de espécies & Número de individuos \\
\hline PARA & 20 & 258 & 3146 \\
\hline ov & 17 & 258 & 3487 \\
\hline BAL & 20 & 271 & 1207 \\
\hline $\mathrm{BC}$ & 26 & 316 & 1962 \\
\hline IRA & 20 & 379 & 1328 \\
\hline Média & 26,60 & 296,60 & 2226 \\
\hline Desvio padrăo & 3,28 & 51,96 & 1042,95 \\
\hline
\end{tabular}

(PARA) Paracatu, Minas Gerais (Projeto de Colonização Mundo Novo); (OV) Formosa do Rio Preto, Bahia (Projeto de Colonização Ouro Verde); (BAL) Balsas, Maranhão (Projeto de Colonização Gerais de Balsas); (BC) Formosa do Rio Preto, Bahia (Projeto de Colonização Brasil Central); (IRA) Iraí de Minas, Minas Gerais (Projeto de Colonização Iraí).

Tabela II. Número de espécies de lepidópteros em cada familia e sua percentagem de contribuição em cinco comunidades ecológicas de Cerrado lato sensu amostradas com armadilha luminosa.

\begin{tabular}{|c|c|c|c|c|c|c|c|c|c|c|}
\hline \multirow{2}{*}{ Familias } & \multicolumn{2}{|c|}{ IRA } & \multicolumn{2}{|c|}{ PARA } & \multicolumn{2}{|c|}{ OV } & \multicolumn{2}{|c|}{$\mathrm{BC}$} & \multicolumn{2}{|c|}{ BAL } \\
\hline & Número & $\%$ & Número & $\%$ & Número & $\%$ & Número & $\%$ & Nimero & $\%$ \\
\hline Agonoxenidae & 0 & 0,00 & 0 & 0,00 & 0 & 0,00 & 1 & 0,32 & 0 & 0,00 \\
\hline Alucitidae & 1 & 0,26 & 0 & 0,00 & 0 & 0,00 & 1 & 0,32 & 1 & 0,37 \\
\hline Apatelodidae & 2 & 0,53 & 2 & 0,75 & 0 & 0,00 & 1 & 0,32 & 2 & 0,74 \\
\hline Arctiidae & 27 & 7,12 & 13 & 5,04 & 9 & 3,49 & 14 & 4,43 & 19 & 7,01 \\
\hline Blastobasidae & 0 & 0,00 & 4 & 1,55 & 1 & 0,39 & 0 & 0,00 & 0 & 0,00 \\
\hline Choreutidae & 5 & 1,32 & 4 & 1,55 & 0 & 0,00 & 0 & 0,00 & 0 & 0,00 \\
\hline Cosmopterigidae & 0 & 0,00 & 1 & 0,39 & 1 & 0,39 & 1 & 0,32 & 0 & 0,00 \\
\hline Cossidae & 1 & 0,26 & 1 & 0,39 & 0 & 0,00 & 1 & 0,32 & 0 & 0,00 \\
\hline Dalceridae & 0 & 0,00 & 2 & 0,75 & 0 & 0,00 & 3 & 0,95 & 1 & 0,37 \\
\hline Epiplemidae & 0 & 0,00 & 0 & 0,00 & 0 & 0,00 & 1 & 0,32 & 2 & 0,74 \\
\hline Gelechiidae & 23 & 6,07 & 16 & 6,20 & 4 & 1,55 & 5 & 1,58 & 2 & 0,74 \\
\hline Geometridae & 38 & 10,03 & 16 & 6,20 & 14 & 5,42 & 25 & 7,91 & 36 & 13,28 \\
\hline Lasiocampidae & 0 & 0,00 & 0 & 0,00 & 0 & 0,00 & 1 & 0,32 & 1 & 0,37 \\
\hline Lecitoceridae & 0 & 0,00 & 0 & 0,00 & 0 & 0,00 & 0 & 0,00 & 1 & 0,37 \\
\hline Limacodidae & 1 & 0,26 & 1 & 0,39 & 2 & 0,77 & 4 & 1,27 & 0 & 0,00 \\
\hline Limantriidae & 0 & 0,00 & 0 & 0,00 & 0 & 0,00 & 0 & 0,00 & 3 & 1,11 \\
\hline Megalopygidae & 3 & 0,79 & 2 & 0,75 & 0 & 0,00 & 2 & 0,63 & 0 & 0,00 \\
\hline Mimallonidae & 2 & 0,53 & 0 & 0,00 & 1 & 0,39 & 0 & 0,00 & 0 & 0,00 \\
\hline Momphidae & 0 & 0,00 & 0 & 0,00 & 1 & 0,39 & 1 & 0,32 & 0 & 0,00 \\
\hline Noctuidae & 126 & 33,24 & 86 & 33,33 & 129 & 50,00 & 135 & 42,72 & 100 & 36,90 \\
\hline Notodontidae & 1 & 0,26 & 5 & 1,94 & 0 & 0,00 & 1 & 0,32 & 4 & 1,47 \\
\hline Oecophoridae & 24 & 6,33 & 4 & 1,55 & 9 & 3.49 & 31 & 9,81 & 15 & 5,53 \\
\hline Oxytenidae & 1 & 0,26 & 0 & 0,00 & 0 & 0,00 & 0 & 0,00 & 0 & 0,00 \\
\hline Psychidae & 0 & 0,00 & 0 & 0,00 & 0 & 0,00 & 2 & 0,63 & 3 & 1.11 \\
\hline Pterophoridae & 2 & 0,53 & 2 & 0,75 & 0 & 0,00 & 1 & 0,32 & 0 & 0,00 \\
\hline Pyralidae & 94 & 24,80 & 73 & 28,29 & 66 & 25,59 & 58 & 18,35 & 60 & 22,14 \\
\hline Saturniidae & 6 & 1,58 & 3 & 1,16 & 4 & 1,55 & 2 & 0,63 & 4 & 1,47 \\
\hline Sphingidae & 7 & 1,85 & 5 & 1,94 & 9 & 3,49 & 10 & 3,16 & 5 & 1,84 \\
\hline Thirididae & 0 & 0,00 & 0 & 0,00 & 2 & 0,77 & 2 & 0,63 & 2 & 0,74 \\
\hline Tineidae & 0 & 0,00 & 7 & 2,71 & 2 & 0,77 & 9 & 2,86 & 9 & 3,33 \\
\hline Tortricidae & 14 & 3,69 & 11 & 4,26 & 3 & 1,16 & 3 & 0,95 & 0 & 0,00 \\
\hline Urodidae & 1 & 0,26 & 0 & 0,00 & 0 & 0,00 & 0 & 0,00 & 0 & 0,00 \\
\hline Yponomeutidae & 0 & 0,00 & 0 & 0,00 & 1 & 0,39 & 1 & 0,32 & 1 & 0,37 \\
\hline Total & 379 & 100,00 & 258 & 100,00 & 258 & 100,00 & 316 & 100,00 & 271 & 100,00 \\
\hline
\end{tabular}

(IRA) Irai de Minas, Minas Gerais; (PARA) Paracatu, Minas Gerais; (OV) Formosa do Rio preto, Bahia; (BC) Formosa do Rio Preto, Bahia; (BAL) Balsas, Maranhão. 


\section{Análises de diversidade e similaridade}

A tabela III mostra os dados de diversidade $(\alpha)$ calculados por dois métodos nas cinco áreas de Cerrado analisadas.

Tabela III. Riqueza de espécies, valores de diversidade $\left(H^{\prime}\right)$ e de diversidade $(\gamma)$ para Lepidoptera em cinco áreas de Cerrado.

\begin{tabular}{|c|c|c|c|}
\hline Localidades & Número de espécies & Diversidade $\left(\mathrm{H}^{\prime}\right)$ & Diversidade $(\gamma)$ \\
\hline PARA & 258 & $1,60 \mathrm{a}$ & $0,42 \mathrm{f}$ \\
\hline ov & 258 & $2,72 \mathrm{~b}$ & $0,72 \mathrm{~g}$ \\
\hline BAL & 271 & $4,59 \mathrm{c}$ & $0,96 \mathrm{~h}$ \\
\hline $\mathrm{BC}$ & 316 & $4,02 d$ & $0,91 i$ \\
\hline IRA & 379 & $4,48 \mathrm{e}$ & $0,93 j$ \\
\hline Total & 296,40 & 3,48 & 0,79 \\
\hline Desvio padrăo & 51,96 & 1,28 & 0,22 \\
\hline
\end{tabular}

(PARA) Paracatu, Minas Gerais; (OV) Formosa do Rio preto, Bahia; (BAL) Balsas, Maranhão; (BC) Formosa do Rio Preto, Bahia; (IRA) Iraí de Minas, Minas Gerais; ( $\left.{ }^{*}\right)$ índice de diversidade de Shannon-Wiener; $\left(^{\star \star}\right)$ indice de diversidade de Simpson. Todos os valores de diversidade acompanhados das letras $(a-j)$ diferem significativamente entre si pelo teste $T(p<0.001)$.

Os valores de diversidade encontrados pelo índice de Shannon-Wiener $\left(\mathrm{H}^{\prime}\right)$ variaram de 1.60 em Paracatu a 4.59 em Balsas. Todas as localidades estudadas são significativamente diferentes entre si pelo teste $T(P<0.001)$. Geralmente estes índices situam-se entre 1.5 e 3.5, raramente ultrapassando 4.5 (MARGALEF 1972; MAGURRAN 1988). Os valores encontrados são altos, principalmente se comparados aos de MARINHO-FILHO et al. (1994), cujos resultados variaram de 0.57 a 3.09 em um estudo de pequenos mamíferos na região dos Cerrados.

Em relação ao índice de diversidade de $\operatorname{Simpson}(\gamma)$, que atribui maior peso para as espécies dominantes, os valores encontrados também foram altos, exceto em Paracatu que apresentou o menor valor (0.42), sendo que o maior valor registrado foi para Balsas (0.96). Por este método também todas as áreas mostraram-se significativamente diferentes pelo teste $\mathrm{T}(\mathrm{p}<0.001)$.

A diversidade beta $(\beta)$ calculada com a finalidade de comparação entre as áreas, apresentou um valor médio de 0.6929 , mínimo de 0.4328 entre Balsas e Formosa do Rio Preto (OV), o que significa áreas bastante semelhantes, e valor máximo de 0.8615 entre Balsas e Iraí de Minas (Tab. IV).

Tabela IV. Valores de diversidade $(\beta)$ e distância em quilômetros entre as cinco áreas de Cerrado estudadas.

\begin{tabular}{|c|c|c|c|c|c|c|}
\hline & \multirow[b]{2}{*}{ Localidades } & \multicolumn{5}{|c|}{ Diversidade beta $(\beta)$} \\
\hline & & PARA & IRA & OV & $B C$ & BAL \\
\hline \multirow{5}{*}{$\begin{array}{l}\text { Distâncias } \\
\qquad(\mathrm{Km})\end{array}$} & PARA & - & 0,6985 & 0,7131 & 0,7526 & 0,8223 \\
\hline & IRA & 200 & - & 0,7017 & 0,7438 & 0,8615 \\
\hline & OV & 650 & 860 & - & 0,5853 & 0,4328 \\
\hline & $\mathrm{BC}$ & 710 & 925 & 65 & - & 0,6183 \\
\hline & BAL & 950 & 1125 & 325 & 265 & - \\
\hline
\end{tabular}

(PARA) Paracatu, Minas Gerais; (IRA) Irai de Minas, Minas Gerais; (OV) Formosa do Rio preto, Bahia; (BC) Formosa do Rio Preto, Bahia; (BAL) Balsas, Maranhão. 
A regressão linear (Fig. 2) entre a distância das áreas consideradas e a diversidade beta, apresentou correlação positiva entre estas variáveis, embora com baixo coeficiente de correlação $\left(\mathrm{r}^{2}=0.5844, \mathrm{p}<0.05\right)$, indicando que a variável distância explica cerca de $60 \%$ das diferenças encontradas.

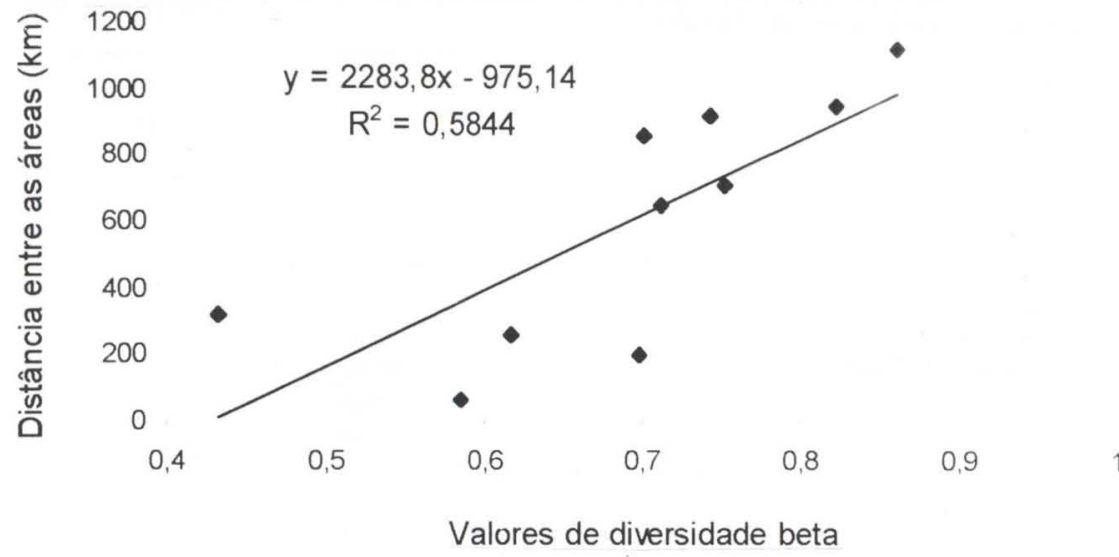

Fig. 2. Regressão linear da distância em função dos valores de diversidade beta para as áreas de Cerrado amostradas, $r^{2}(p<0.05)$.

Para avaliação da similaridade na composição de espécies, entre as diferentes áreas pesquisadas, foram utilizados dois índices qualitativos (de Sörensen e de Jaccard), ambos baseados na presença e na ausência de espécies. Pelos dois métodos utilizados foram encontrados baixos valores de semelhança (Tab. V). Normalmente uma similaridade acima de 0.5 tem sido considerada alta por alguns autores (FELFILI et al. 1994).

Tabela V. Análise comparativa da composição de espécies através de dois índices de similaridade nas áreas de Cerrado estudadas.

\begin{tabular}{clccccc}
\hline \multirow{2}{*}{ Indices } & & \multicolumn{5}{c}{ Sörensen } \\
\cline { 3 - 7 } & Localidades & PARA & IRA & OV & BC & BAL \\
\hline \multirow{2}{*}{ Jaccard } & PARA & - & 0,3014 & 0,2868 & 0,2473 & 0,1776 \\
& IRA & 0,1774 & - & 0,2982 & 0,2561 & 0,1384 \\
& OV & 0,1674 & 0,1752 & - & 0,4146 & 0,3742 \\
& BAL & 0,1411 & 0,1468 & 0,2615 & - & 0,3902 \\
\end{tabular}

(PARA) Paracatu, Minas Gerais; (IRA) Irai de Minas, Minas Gerais; (OV)Formosa do Rio preto, Bahia; (BC) Formosa do Rio Preto, Bahia; (BAL) Balsas, Maranhão.

Pelo índice de Sörensen o menor valor de semelhança encontrado foi de 0.1384 entre Balsas e Iraí de Minas, e o maior valor foi de 0.4146 entre as duas áreas localizadas em Formosa do Rio Preto, no oeste do estado da Bahia.

Pelo método de Jaccard, os índices de semelhanças apresentam-se menores do que pelo método anterior. Entretanto, as localidades com menor valor $(0.0743)$ e maior valor (0.2615) são as mesmas detectadas pelo método anterior. 
De maneira geral, o número de espécies compartilhadas entre os pares de localidades, e também entre as cinco áreas amostradas foi baixo. Apenas $11.4 \%$ das espécies estiveram representadas em todos os locais.

Os resultados de compartilhamento devem ser analisados considerando que, embora o esforço de captura empregado e outras condições de amostragem tenham sido as mesmas para as cinco áreas, existem flutuações anuais e sazonais ainda pouco compreendidas para o grupo, além disso, os dados representam uma amostra das comunidades estudadas.

\section{Dominância}

A freqüência de espécies relacionada ao número de indivíduos (equitabilidade) é um componente da diversidade que mostra a existência ou não de dominância. Somente pode-se falar.de dominância em comunidades de baixa diversidade, visto que, geralmente existe correlação negativa entre as duas (MARGALEF 1991). Estudos sobre comunidades tropicais sugerem que a maioria das espécies apresentem poucos indivíduos (PRESTON 1948, 1960). Esta tendência foi observada nas cinco áreas estudadas onde a maioria das espécies estiveram representadas por apenas um indivíduo (Fig. 3).
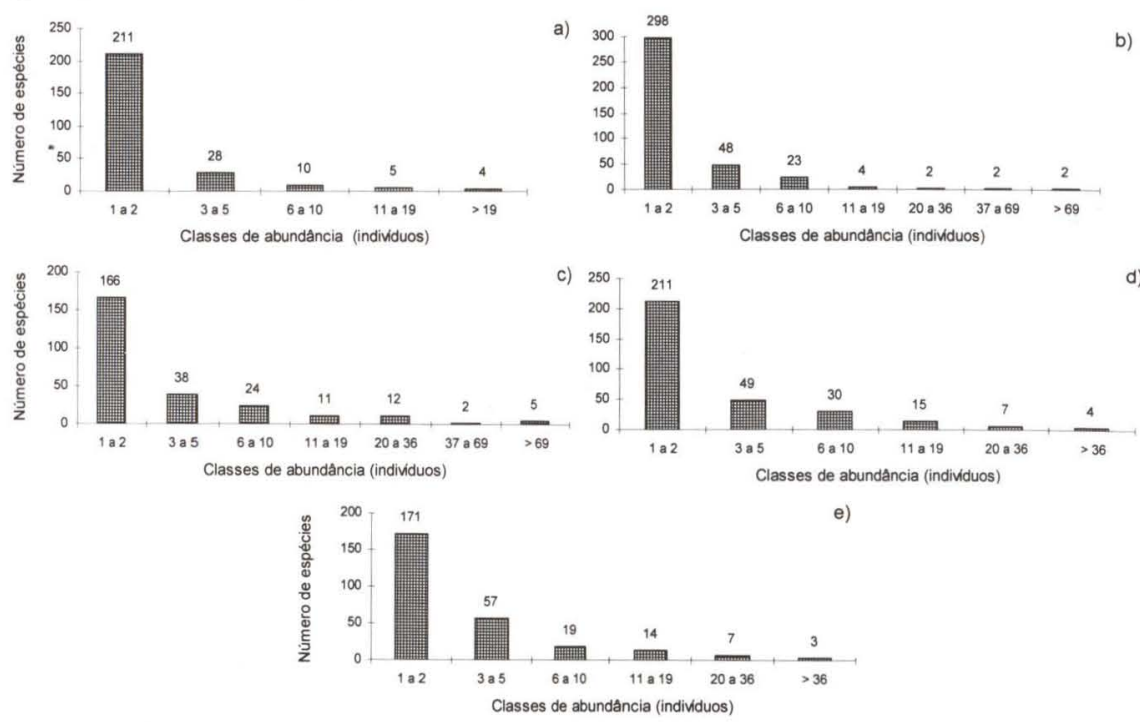

Fig. 3. Freqüência de espécies relacionada ao número de indivíduos em cinco áreas da região dos Cerrados: (a) Paracatu, Minas Gerais; (b) Iraí de Minas, Minas Gerais; (c) Formosa do Rio Preto, Bahia (Projeto de Colonização Ouro Verde); (d) Formosa do Rio Preto, Bahia (Projeto de Colonização Brasil Central); (e) Balsas, Maranhão.

\section{CONCLUSÕES}

A fauna de lepidópteros noturnos apresenta-se distribuída diferenciadamente entre as famílias, sendo que Noctuidae e Pyralidae são as famílias que apresentaram maior percentagem de contribuição nas cinco comunidades estudadas. 
Observou-se baixa dominância em todas as áreas, isto é, a maioria das espécies estiveram representadas por até dois indivíduos.

De maneira geral, os valores de diversidade alfa encontrados foram altos e todas as áreas comparadas por este parâmetro são significativamente diferentes entre si $(\mathrm{p}<0.001)$.

Foi observada grande variação nos valores de diversidade beta entre as áreas, sendo constatada uma maior afinidade na composição de espécies entre Balsas, MA e uma das áreas da Bahia (Projeto Ouro Verde). As localidades com menor semelhança foram Balsas, Maranhão e Iraí de Minas, Minas Gerais. A regressão linear da distância em função da diversidade beta apresentou correlação positiva entre estas variáveis $\left(\mathrm{r}^{2}=0.5844, \mathrm{p}<0.05\right)$.

Foram constatados baixos níveis de similaridade entre os locais estudados. Pelo índice de Sörensen os valores variaram de 0.1384 entre Balsas e Iraí de Minas, e 0.4146 entre as duas áreas de Formosa do Rio Preto, Bahia. Pelo índice de Jaccard, as áreas com menor similaridade $(0,0743)$ e maior similaridade $(0,2615)$ foram as mesmas encontradas pelo método anterior.

AGRADECIMENTOS. À Companhia de Promoção Agrícola (CAMPO) e à Japan International Cooperation Agency (JICA) por terem financiado este estudo. Às Cooperativas Agrícolas: COTIA; COPAMIL; COACERAL e BATAVO, pelo fornecimento das estruturas de campo durante as coletas. Ao Dr. Vítor O. Becker pelo auxílio na identificação do material. Aos Srs. Wellington Cavalcanti, Joaquim Afonso P. Maciel, João Batista dos Santos, Antônio Lisboa de L. Gomes e Marcos S. Ferreira (Embrapa Cerrados), pelo auxílio nos trabalhos de campo e laboratório.

\section{REFERÊNCIAS BIBLIOGRÁFICAS}

AB'Saber, A.N. 1977. Os domínios morfoclimáticos da América do Sul. Primeira aproximação. Geomorfologia 52: 1-21.

1983. O domínio dos Cerrados: uma introdução ao conhecimento. Revta Serv. Púb., Brasília, 40 (111): 41-55.

AdÁmoli, J.; J. MACEDO; L.G. AZEVEdo \& J.MAdeira Neto. 1986. Caracterização da região dos Cerrados, p.33-74. In: W.J. GoEDERT (Ed.). Solos dos Cerrados: tecnologias e estratégias de manejo. São Paulo, Nobel e Brasília, Embrapa, XV+422p.

AlHo, C.J.R. 1994. Distribuição da Fauna num Gradiente de Recursos em Mosaico, p.213-262. In: M.N. PINTO (Ed.). Cerrado: caracterização, ocupação e perspectivas. Brasília, Sematec, Univ. de Brasília, XXI+657p.

BROWN, K.S. JR. \& O.H.H. MielKE. 1967a. Lepidoptera of the Central Brazil Plateau. I. Preliminary list of Rhopalocera: Introduction, Nymphalidae, Libytheidae. Jour. Lep. Soc. 21 (2): 77-106.

. 1967b. Lepidoptera of the Central Brazil Plateau. I. Preliminary list of Rhopalocera (continued): Lycaenidae, Pieridae, Papilionidae, Hesperiidae. Jour. Lep. Soc. 21 (3): 145-168.

Costa, C.C.C.; J.P. Lima; L.D. CARDoso \& V.Q. HenRiQues. 1981. Fauna do Cerrado: lista preliminar de aves, mamíferos e répteis. Rio de Janeiro, IBGE, $\mathrm{V}+189 \mathrm{p}$. 
DiAS, B.F. DE S. 1992. Cerrados: Uma caracterização, p.7-26. In: B.F. DE S. DiAS (Ed.). Alternativas de Desenvolvimento dos Cerrados: Manejo e Conservação dos Recursos Naturais Renováveis. Brasília, Univ. Brasília, Ibama, Funatura, I+97p.

Felfili, M.J.; M. Haridassan; R.C. MendonÇA; T.S. Filgueiras; M.C. DA Silva JR. \& A.V. RezEndE. 1994. Projeto biogeografia do bioma Cerrado: vegetação e solos. Caderno de Geociências, Rio de Janeiro, 12: 75-166.

JACCARD, P. 1912. The distribution of the flora in the alpine zone. Nerophytal 11: 37-50.

MagurRan, A.E. 1988. Ecological Diversity and its measurement. Princeton, New jersey, VI+179p.

MARGALEF, R. 1972. Homage to Evelyn Hutchinson, or why is there an upper limit to diversity. Trans. Connect. Acad. Arts Sci. 44: 211-235.

1991. Ecologia. Barcelona, Omega, XXVII+951p.

Marinho-Filho, J.; M.L. Reis; P.S. Oliveira; E.M. Vieira \& M.V. PAes. 1994. Diversity standards and small mammal numbers: conservation of the cerrado biodiversity. An. Acad. Brasil. Ciêncais 66 (Supl. 1): 149-157.

MARINHO-FILHO, J.S. 1992. Biogeografia, p.65-68. In: B.F. DE S. DiAS (Ed.). Alternativas de Desenvolvimento dos Cerrados: Manejo e Conservação dos Recursos Naturais Renováveis. Brasília, Univ. Brasília, Ibama, Funatura, $\mathrm{V}+97 \mathrm{p}$.

MielKe, O.H.H. 1968. Lepidoptera of the Central Brazil Plateau. II. New genera, species, and subspecies of Hesperiidae. Jour. Lep. Soc. 22 (1): 1-20.

PinHEIRO, C.E.G. \& J.V.C. ORTiz. 1992. Communities of fruit-feeding butterflies along a vegetation gradient in central Brazil. Jour. Biogeog., Oxford, 19: 505-511.

Preston, F.W. 1948. The commonness and rarity of species. Ecology 29: 254-283. . 1960. Time and space and the variation of species. Ecology 41: 611-627.

RATTER, J. \& T.C. DARGIE. 1992. An analysis of the floristic composition of 26 cerrado areas in Brazil. Royal Botanic Garden of Edinburg 49 (2): 235-250.

RATTER, J.; S. BRIDGEWATER; R. ATKINSON \& J.F. RIBEIRO. 1996. Analysis of the floristic composition of the Brazilian cerrado vegetation II: comparison of the woody vegetation of 98 areas. Royal Botanic Garden of Edinburg 53 (2): 153-180.

REDFORD, K. H. \& G.A.B. FonsECA. 1986. The role of gallery forests in the zoogeography of the cerrado's non-volant mammalian fauna. Biotropica 18: $126-135$.

RiBEIRO, J.F. \& J.C.S. SiLVA. 1996. Manutenção e recuperação da biodiversidade do bioma Cerrado: o uso de plantas nativas, p.10-14. In: Anais of the $1^{\text {st }}$ International Symposium on Tropical Savannas and VIII. Simpósio sobre o Cerrado, Brasília. Brasília, Centro de Pesquisa Agropecuária dos CerradosCPAC, Embrapa, 508p.

Sick, H. 1965. A fauna do cerrado. Arq. Zool., São Paulo, 12: 71-93.

SILVA, J.M. DA. 1995a. Biogeographic analysis of the South American Cerrado avifauna. Steenstrupia 21: 49-67.

1995b. Birds of the Cerrado Region, South America. Steenstrupia 21: 
69-92.

Silveira Neto, S.; O. Nakano; D. Bahiarbin \& N.A. Villa Nova. 1976.

Manual de ecologia dos insetos. São Paulo, Agonômica Ceres, VI+419p.

SöRENSEN, T. 1948. A method of establishing groups of equal amplitude in plant sociology based an similarity of species. K. Danske Videnske Selsk 5: 1-34.

ThOMAs, A.W. \& G.M. ThOMAS. 1994. Sampling strategies for estimating moth species diversity using a light trap in a northeastern softwood forest. Jour. Lep. Soc. 48 (2): 95-105.

Vanzolini, P.E. 1963. Problemas faunísticos do Cerrado, p.305-321. In: M.G. FERrI (Ed.). Simpósio sobre o Cerrado. São Paulo, Univ. de São Paulo, $\mathrm{X}+424 \mathrm{p}$.

WHITTAKER, R.H. 1960. Vegetation of the Siskiyou mountains, Oregon and California. Ecol. Monogr. 30: 279-338.

Recebido em 31.X.1997; aceito em 22.III.1999. 